\title{
POTENTIAL INAPPROPRIATE USE OF STRONG OPIOID ANALGESICS IN CANCER OUTPATIENTS DURING THE LAST YEAR OF LIFE IN FRANCE AND ASSOCIATED FACTORS
}

\author{
Thanh Hang CHU ${ }^{1}$, Manuela Rueter ${ }^{2}$, Aurore Palmaro ${ }^{1}$, and Maryse Lapeyre-Mestre ${ }^{1}$ \\ ${ }^{1}$ Université Toulouse III Paul Sabatier \\ ${ }^{2}$ INSERM
}

June 1, 2021

\begin{abstract}
Aim: A better knowledge of opioid prescribing patterns would help to identify areas of potential improvement in cancer pain management. This study aimed to identify potential inappropriate use (PIU) of strong opioid analgesics in cancer outpatients in their last year of life. Methods: A retrospective cohort of cancer patients dead between 2011 and 2014 and who were exposed as outpatient to a strong opioid analgesic in the last year of life was identified in the "Echantillon Généraliste de Bénéficiaires" (a 1/97th random sample of the French general population). Prescribing patterns of strong opioids were analyzed and PIU was defined by at least one of these criteria: overlapping prescriptions; contraindicated prescriptions; lack of laxatives; potential drug interactions; prescription in patients hospitalized for opioid-related disorders. Factors associated with PIU were investigated through a multiple logistic regression model. Results: One third of the 2,236 patients (median age 72 years (IQR: 61-82), $44.1 \%$ of women) presented a PIU (insufficient laxative prescription (19.6\% of patients), insufficient background treatment with transmucosal fentanyl (14.8\%), overlapping prescriptions (2.6\%)). The rate of PIU significantly decreased from $37.6 \%$ (2011) to $29.8 \%$ (2014). For patients with a duration of opioid use ? 3 months, factors associated with PIU were fentanyl prescription $(\mathrm{aOR}=2.36 ; 95 \% \mathrm{CI}[1.86-3.00])$ and previous use of strong opioid $(\mathrm{aOR}=1.88 ;$ [1.50-2.36]) Conclusion: In France, one third of cancer patients exposed to strong opioids experienced PIU and this proportion tended to decrease over time. There is still room for progress in cancer pain management at the end of life.
\end{abstract}

\section{Hosted file}

Opioid EOL BJCP 2021-2904.docx available at https://authorea.com/users/417325/articles/ 524464-potential-inappropriate-use-of-strong-opioid-analgesics-in-cancer-outpatientsduring-the-last-year-of-life-in-france-and-associated-factors 